\title{
ANÁLISE FLORÍSTICA E ESTRUTURAL DE FLORESTAS RIPÁRIAS DO ALTO RIO PARANÁ, TAQUARUÇU, MS ${ }^{1}$
}

\author{
Mariza Barion Romagnolo ${ }^{2}$ \\ Maria Conceição de Souza ${ }^{3}$
}

Recebido em 12/01/1999. Aceito em 22/02/2000

\begin{abstract}
RESUMO - (Análise florística e estrutural de florestas ripárias do Alto Rio Paraná, Taquaruçu, MS). Utilizandose de método fitossociológico, foi realizado estudo comparativo entre três remanescentes de floresta ripária da margem direita do alto rio Paraná $\left(22^{\circ} 43^{\prime}-45^{\prime} \mathrm{S} ; 53^{\circ} 15^{\prime}-18^{\prime} \mathrm{W}\right)$. Em cada um deles foi demarcada uma área de $3.150 \mathrm{~m}^{2}$, subdividida em 21 parcelas contínuas de $150 \mathrm{~m}^{2}$, tendo como critério mínimo de amostragem o perímetro à altura do peito igual ou superior a $15 \mathrm{~cm}$. Foram levantados, na amostragem total, 58 espécies, 46 gêneros e 30 famílias, sendo duas indeterminadas. As famílias de maior riqueza específica foram Myrtaceae e Lauraceae com cinco espécies cada, Mimosaceae com quatro, Annonaceae e Rubiaceae com três cada uma, enquanto que os gêneros foram, Nectandra com quatro, Eugenia, Inga, Pouteria, Psidium e Sloanea com dois cada um. Os índices de diversidade (H') específicos foram de 2,94, 2,52 e 2,88 para, respectivamente, os remanescentes jusante, intermediário e montante. O índice de similaridade de Sørensen entre os três remanescentes variou de $54 \%$ a $65 \%$. Do total de 58 espécies amostradas, somente $16(26,7 \%)$ ocorreram nos três remanescentes. Dentre as 10 espécies de maior valor de importância, apenas Cecropia pachystachya e Inga uruguensis foram comuns aos remanescentes. Os resultados da análise fitossociológica, principalmente, permitiram concluir que o remanescente intermediário encontra-se em fase mais imatura de sucessão. A homogeneidade da topografia e do solo indicam que as diferenças estruturais entre os três remanescentes devem-se a fatores relacionados à antropização.
\end{abstract}

Palavras-chave - florística, fitossociologia, mata ciliar, floresta aluvial, rio Paraná, planície de inundação

ABSTRACT - (Floristic and structural analyses of riparian forest of the "Alto Rio Paraná", Taquaruçu, MS). In
this study, the structure of the vegetation and the species diversity of three remnants of riparian forest of the right
bank of the upper Paraná river $\left(22^{\circ} 43^{\prime}-45^{\prime} \mathrm{S} ; 53^{\circ} 15^{\prime}-18^{\prime} \mathrm{W}\right.$ ) were analysed and compared. In each forest remnant,
$3,150 \mathrm{~m}^{2}$ were marked and subdivided into 21 continuous plots of $150 \mathrm{~m}^{2}$ each. Only trees with a perimeter at breast
height $\geq 15 \mathrm{~cm}$ were measured. Fifity eigth species belonging to 46 genera and 30 families were found. Two taxa at
the family level were still unidentified. The families Myrtaceae and Lauraceae had the highest number of species
(five each), followed by Mimosaceae (four), Annonaceae and Rubiaceae (three each). The richest genera in terms
of number of species were Nectandra, four, and Eugenia, Inga, Pouteria, Psidium and Sloanea, with two each. The
specific diversity index for the downstream, the intermediate, and the upstream site were respectively $2.94,2.52$

1 Parte da Dissertação de Mestrado do primeiro Autor; trabalho apresentado no $49^{\circ}$ Congresso Nacional de Botânica, Salvador, BA, 1998

2 Pós-graduação em Ecologia de Ambientes Aquáticos Continentais, Universidade Estadual de Maringá, Departamento de Biologia, Nupelia, Maringa, PR, Brasil

3 Universidade Estadual de Maringá, Departamento de Biologia, Nupelia, Avenida Colombo, 5790, CEP 87020-900, Maringá, PR, Brasil 
and 2.88. The Sørensen similarity index was high between sites, ranging from $54 \%$ to $65 \%$. Only $16(26.7 \%)$ of the total of the species occurred in the three sites, the remaining species occurring in either one or two sites. Out of the 10 species with the highest importance value, only Cecropia pachystachya and Inga uruguensis were common in the three sites. The phytosociological analysis demonstrated that the intermediate remnant is in a less mature successional stage. The homogeneity of topography and soil indicated that the structural differences between the remnants might be related to anthropic action.

Key words - floristic, phytosociology, riparian forest, alluvial forest, Paraná river, floodplain

\section{Introdução}

A vegetação ripária, com sua distribuição restrita às faixas de contato entre os ambientes terrestre e aquático, atua regulando os processos de entrada de luz e nutrientes no sistema aquático, servindo de barreira física e auxiliando na recarga dos aqüíferos subterrâneos (Elmore 1992) e mantendo diversas relações com a fauna aquática e terrestre. Caracterizase, desta forma, como um ecótono água-terra (Holland \& Risser 1988; Zalewski et al. 1990), sendo de extrema importância sua preservação e recuperação.

A planície de inundação do alto rio Paraná, último trecho livre de barragens desse rio, apresenta diferentes tipos de formações vegetais ripárias, tais como Floresta Estacional Semidecidual Submontana, Floresta Estacional Semidecidual Aluvial e formações de campo representadas pelas várzeas e áreas antropizadas, principalmente para pastagem (Campos \& Souza 1997).

Levantamentos florísticos e fitossociológicos, para caracterização da vegetação dessa região, foram desenvolvidos por Assis (1991), Romagnolo et al. (1994), Souza-Stevaux et al. (1994), Silva (1995), Campos \& Souza (1997), Campos et al. (1997), Souza et al. (1997), Souza (1998) e Souza \& Souza (1998). A heterogeneidade florística e fitossociológica, demonstrada por esses trabalhos, de acordo com Souza et al. (1997) indica, no entanto, a necessidade de haver continuidade desses estudos, buscando-se ampliar a área de amostragem e abranger maior número de ambientes e de fases de maturação da floresta.
O presente estudo, neste contexto, teve como objetivo proceder à análise comparativa da florística e da estrutura fitossociológica da vegetação arbustivo-arbórea, em três remanescentes de floresta ripária na planície de inundação do alto rio Paraná.

\section{Material e métodos}

Área de estudo - A área de estudo localizase na margem direita do alto rio Paraná $\left(22^{\circ} 43^{\prime}-\right.$ $45^{\prime}$ S; $53^{\circ} 15^{\prime}-18^{\prime} \mathrm{W}$ ), Município de Taquaruçu, Estado de Mato Grosso do Sul (Fig. 1). O rio Paraná apresenta, neste trecho, amplo canal anastomosado, com baixa declividade e planície de inundação que se desenvolve em toda sua margem direita, abrigando diversos subambientes fluviais, tais como pântanos, lagoas, canais secundários e diques marginais que são de grande importância para a ecologia local (Stevaux 1994; Agostinho \& Zalewski 1996). Com altitude variando de 200 a 300m, essa área enquadra-se no período Quaternário da era Cenozóica (Stevaux 1994). O clima é do tipo subtropical úmido com verão quente (temperatura média mais quente acima de $22^{\circ} \mathrm{C}$ ) e índice pluviométrico médio anual entre $1.200 \mathrm{e}$ $1.300 \mathrm{~mm}$, de acordo com o Sistema de Köppen, e as chuvas distribuem-se durante todos os meses do ano, com maior volume no período de setembro a dezembro e com as menores médias nos meses de junho a agosto, porém sempre superiores a 30mm (IAPAR 1994).

A cobertura vegetal encontra-se, de acordo com Veloso \& Góes-Filho (1982), sob domínio da Floresta Estacional Semidecidual Submontana sendo que, principalmente na mar- 


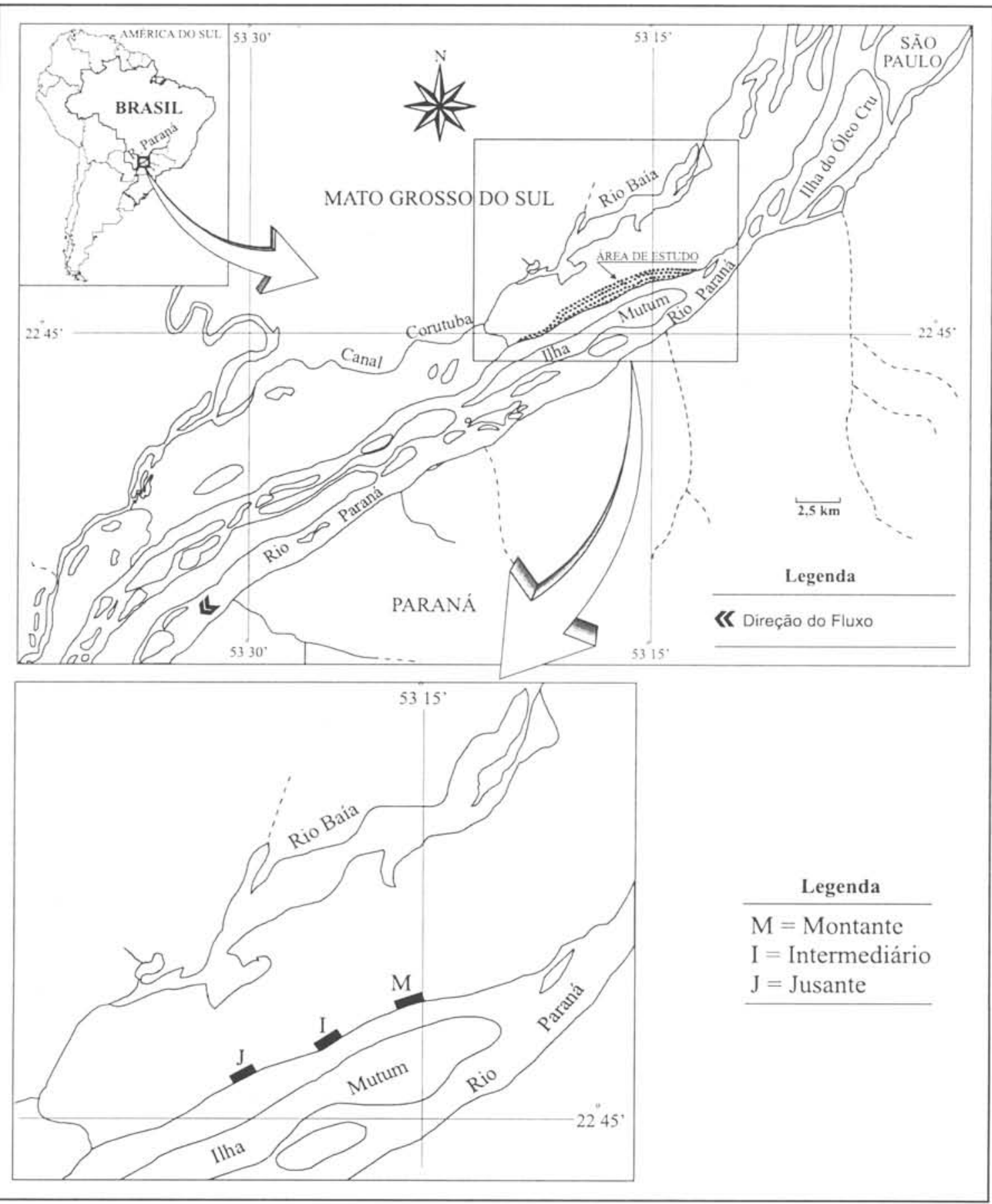

Figura 1. Localização da área de estudo. Acima: vista geral. Abaixo: localizaçâo dos três remanescentes de floresta ripária analisados neste estudo (Município Taquaruçu. MS). 
gem direita, local deste estudo, ocorre a Floresta Estacional Semidecidual Aluvial (Campos \& Souza 1997), além da qual há a área de várzea que se estende até as margens do rio Baía. Essas florestas, sujeitas a inundação durante as cheias mais pronunciadas, encontram-se, atualmente, em diferentes graus de sucessão, resultantes, principalmente, de práticas agropecuárias desenvolvidas na região.

Para este estudo foram escolhidos três remanescentes marginais da Floresta Estacional Semidecidual Aluvial, denominados montante, intermediário e jusante (Fig. 1). Estes se assemelhavam quanto à topografia e à exposição às inundações esporádicas que ocorrem nas cheias mais pronunciadas. Os remanescentes eram eqüidistantes em aproximadamente $1.500 \mathrm{~m}$, sendo intercalados por áreas abertas, resultantes de pastagem e com esparsos indivíduos arbóreos de espécies heliófitas, como Cecropia pachystachya, Croton urucurana e Inga uruguensis.

Perfil topográfico e análise do solo - O perfil topográfico, para cada remanescente, foi obtido pelo método da variação do nível, utilizando-se uma mangueira transparente com água acoplada a régua graduada (Godoy 1988).

Para a análise do solo, foram coletadas em cada área três amostras individualizadas, nas profundidades de $0-30 \mathrm{~cm}$ e de $30-90 \mathrm{~cm}$, de acordo com EMBRAPA (1984). As análises químicas e granulométricas foram realizadas pelo Laboratório de Análise de Solos da Universidade Estadual de Maringá. Para comparar as três áreas amostradas, aplicou-se análise de variância multivariada (Johnson \& Wichern 1992).

Florística e fitossociologia - Em cada remanescente foi delimitada uma área de $3.150 \mathrm{~m}^{2}$ ( $105 \mathrm{~m}$ paralelos $\mathrm{x} 30 \mathrm{~m}$ perpendiculares ao eixo do rio), totalizando $9.450 \mathrm{~m}^{2}$. Cada uma foi subdividida em 21 parcelas contínuas de $150 \mathrm{~m}^{2}$ cada ( $15 \mathrm{~m}$ paralelos $\times 10 \mathrm{~m}$ perpendiculares), onde foram amostrados indivíduos com perímetro do caule, a 1,30m de altura (PAP), igual ou superior a $15 \mathrm{~cm}$. Indivíduos ramificados abaixo dessa altura somente foram amostrados se pelo menos um dos ramos apresentasse o PAP estabelecido. Considerando-se que as árvores mortas e em pé constituem indicativo da dinâmica sucessional da floresta (Martins 1979), esses indivíduos foram também amostrados e, devido à dificuldade na identificação taxonômica desse componente estrutural, incluídos num único grupo denominado "morta". Cada indivíduo amostrado recebeu uma plaqueta numerada e teve o PAP medido. Simultaneamente, foram coletadas amostras para herborização, de acordo com técnicas usuais (Fidalgo \& Bononi 1989) e incorporadas ao acervo do Herbário da Universidade Estadual de Maringá (HUM). A identificação das espécies foi realizada por consulta à literatura especializada e comparação às exsicatas do herbário acima citado e do Museu Botânico Municipal de Curitiba (MBM). A análise fitossociológica foi realizada pelo programa FITOPAC ${ }^{\circledR}$ versão 1.4 , que forneceu os parâmetros fitossociológicos e os índices comumente obtidos nesses levantamentos (Müller-Dombois \& Ellenberg 1974; Martins 1979; Matteucci \& Colma 1982; Rodrigues 1989), como Freqüência, Densidade e Dominância Absolutas e Relativas; Valor de Importância (VI) e Índice de Diversidade de Shannon (H'). A comparação da similaridade florística entre as três áreas foi feita pelo Índice de Similaridade de Sørensen, que expressa a semelhança entre duas áreas, baseando-se no número de espécies comuns (Müller-Dombois \& Ellenberg 1974).

\section{Resultados e discussão}

Perfil topográfico e análise do solo - O perfil topográfico dos três remanescentes analisados apresenta-se plano, com declividade média de $3 \%$, da margem para o interior. A topografia da margem representa um dos principais fatores que afetam a composição florística de áreas ripárias. Sua morfologia expõe a vegetação, em maior ou menor grau, à variação do nível do len- 
çol freático e à inundação, determinando as espécies vegetais adaptadas a ocuparem essas áreas (Rodrigues 1989).

O solo dos três remanescentes, classificado de acordo com EMBRAPA (1984), é do tipo aluvial não hidromórfico, pouco desenvolvido e derivado de sedimentos aluviais não consolidados, possuindo o horizonte A diferenciado e assente sobre camadas usualmente estratificadas, sem relação pedogenética e composição quími$\mathrm{ca}$, mineralógica e granulométrica variadas. Aplicando-se Análise de Variância Multivariada (MANOVA: $\mathrm{p}<0,05 ; \mathrm{n}_{30 \mathrm{~cm}}=9 ; \mathrm{n} 90 \mathrm{~cm}=9$ ), para os resultados das análises granulométricas, químicas e de $\mathrm{pH}$ (Tab. 1), verifica-se que não há diferença estatisticamente significativa entre os três remanescentes e as duas profundidades analisadas. A fertilidade e a textura do solo constituem, também, importantes fatores condicionantes da vegetação de áreas ripárias. Sua textura determina a disponibilidade de oxigênio e retenção de água, que são altamente afetados pelas inundações (Mantovani 1989; Rodrigues 1989).

Tendo-se em vista a importância da topografia e do solo na determinação da vegetação de áreas ripárias, verifica-se que os remanescentes estudados encontram-se sob influência semelhante, resultante da homogeneidade desses fatores.

Florística e fitossociologia - Nos $9.450 \mathrm{~m}^{2}$ delimitados para este levantamento, foram amostrados 1.877 indivíduos, dos quais 125
(7,0\%) pertencem à categoria morta. Os indivíduos restantes distribuem-se em 58 espécies, 46 gêneros e 30 famílias, sendo que duas destas encontram-se indeterminadas devido à ausência de material reprodutivo e à raridade na região. As famílias de maior riqueza específica são Myrtaceae e Lauraceae, com cinco espécies cada, Mimosaceae com quatro e Annonaceae e Rubiaceae com três cada uma, enquanto que os gêneros são Nectandra, com quatro e Eugenia, Inga, Pouteria, Psidium e Sloanea com dois cada um. Cinqüenta por cento das famílias estão presentes nos três remanescentes, $33,3 \%$ em dois e $16,7 \%$ em apenas um deles enquanto que, dentre as espécies, $26,7 \%$ são comuns para os três, $35,70 \%$ para dois e $37,50 \%$ são exclusivas de apenas um. Dentre as espécies exclusivas, nove o são para o trecho montante, oito para o jusante e quatro para o intermediário (Tab. 2), sendo que dessas apenas Nectandra cuspidata, N. mollis, Psidium guineense e as indeterminadas 1 e 2 são, até o momento, de ocorrência exclusiva para este levantamento; Citrus aurantium, Guatteria sp., Maclura tinctoria e Xilosma venosum ocorrem em apenas um, e as demais ocorrem entre dois a cinco desses levantamentos já realizados nesta região (Campos 1997; Souza et al. 1997).

A similaridade florística entre esses remanescentes pode ser verificada pelo Índice de Similaridade de Sørensen que é de $65,3 \%$, entre montante e jusante, $63,2 \%$ entre intermediário e jusante e de $54,2 \%$ entre montante e intermediário. Esses valores encontram-se entre os mais

Tabela 1. Análise física e química do solo, (média \pm desvio padrão, $\mathrm{n}=3$ ) coletado nas profundidades de $0-30$ e $30-90 \mathrm{~cm}$ nos remanescentes de floresta ripária da margem direita do alto rio Paraná (Município Taquaruçu, MS). (M = montante, $\mathrm{I}=$ intermediário, $\mathrm{J}=$ jusante, $\mathrm{AG}=$ areia grossa, $\mathrm{AF}=$ areia fina, $\mathrm{Sil}=$ silte, $\mathrm{Arg}=\operatorname{argila}$ ).

\begin{tabular}{|c|c|c|c|c|c|c|c|c|c|c|c|c|c|}
\hline \multirow[b]{3}{*}{ Local } & \multirow{3}{*}{$\begin{array}{l}\text { Prof. } \\
(\mathrm{cm})\end{array}$} & \multirow{2}{*}{\multicolumn{4}{|c|}{$\begin{array}{l}\text { Análise granulométrica } \\
(\%)\end{array}$}} & \multirow[b]{3}{*}{$\mathrm{pH}$} & \multicolumn{7}{|c|}{ Análise de Fertilidade } \\
\hline & & & & & & & \multicolumn{5}{|c|}{ meq $/ 100 \mathrm{ml}$ de solo } & \multirow{2}{*}{$\frac{\mathrm{ppm}}{\mathrm{P}}$} & \multirow{2}{*}{ \% } \\
\hline & & AG & $\mathrm{AF}$ & Sil & Arg & & $\mathrm{Al}$ & HAI & $\mathrm{Ca}$ & $\mathrm{Mg}$ & $\mathrm{K}$ & & \\
\hline \multirow[t]{2}{*}{ M } & $0-30$ & $01 \pm 0$ & $48 \pm 16$ & $24 \pm 11$ & $26 \pm 4$ & $5,0 \pm 0,05$ & $0,53 \pm 0,15$ & $3,81 \pm 1,11$ & $3,44 \pm 0,69$ & $1,03 \pm 0,16$ & $0,21 \pm 0,09$ & $3 \pm 1$ & $14,94 \pm 0.58$ \\
\hline & $30-90$ & $01 \pm 0$ & $61 \pm 9$ & $15 \pm 6$ & $23 \pm 3$ & $4,9 \pm 0,15$ & $0,70 \pm 0,53$ & $4,50 \pm 0,48$ & $2,17 \pm 0,31$ & $0,82 \pm 0.12$ & $0,08 \pm 0.01$ & $2 \pm 1$ & $7,60 \pm 1,74$ \\
\hline \multirow[t]{2}{*}{ I } & $0-30$ & $01 \pm 0$ & $46 \pm 5$ & $24 \pm 2$ & $29 \pm 4$ & $5,2 \pm 0,15$ & $0,20 \pm 0,15$ & $3,37 \pm 2,55$ & $5.15 \pm 1.25$ & $1,34 \pm 0,32$ & $0,15 \pm 0.01$ & $2 \pm 0,5$ & $16,45 \pm 5,96$ \\
\hline & $30-90$ & $01 \pm 0$ & $73 \pm 5$ & $07 \pm 3$ & $19 \pm 2$ & $4,9 \pm 0,32$ & $1,00 \pm 0,41$ & $5,04 \pm 1,12$ & $2,26 \pm 0,54$ & $0,87 \pm 0,04$ & $0.08 \pm 0,01$ & $4 \pm 1$ & $7,08 \pm 0,58$ \\
\hline \multirow[t]{2}{*}{ J } & $0-30$ & $01 \pm 0$ & $71 \pm 3$ & $12 \pm 1$ & $16 \pm 3$ & $5,4 \pm 0,30$ & $0,10 \pm 0,05$ & $4,30 \pm 1,75$ & $4,83 \pm 1,26$ & $1,25 \pm 0,40$ & $0.17 \pm 0.07$ & $6 \pm 1$ & $12,54 \pm 2,28$ \\
\hline & $30-90$ & $01 \pm 0$ & $69 \pm 16$ & $11 \pm 9$ & $19 \pm 6$ & $5,4 \pm 0.25$ & $0.10 \pm 0.15$ & $7.20 \pm 2.15$ & $3.10 \pm 0.90$ & $0,88 \pm 0,13$ & $0,10 \pm 0,03$ & $4 \pm 0$ & $7,42 \pm 0,84$ \\
\hline
\end{tabular}


Tabela 2. Relação das famílias, gêneros e espécies arbustivo-arbóreas ( $\mathrm{PAP} \geq 15 \mathrm{~cm}$ ) com respectivas ocorrências entre os três remanescentes estudados $(\mathrm{M}=$ montante, $\mathrm{I}=$ intermediário, $\mathrm{J}=$ jusante) e número de registro de herbário (HUM). (Município de Taquaruçu, MS).

\begin{tabular}{|c|c|c|c|c|}
\hline Famílias & Nome científico & Nome vulgar & Local & HUM \\
\hline Anacardiaceae & Spondias Iutea L. & cajá & $\mathrm{J}, \mathrm{I}, \mathrm{M}$ & 3978 \\
\hline \multirow[t]{3}{*}{ Annonaceae } & Guatteria sp. & & $\mathrm{J}$ & 3993 \\
\hline & Rollinia emarginata Schl. & ariticum-do-mato & $\mathrm{J}, \mathrm{I}, \mathrm{M}$ & 3991 \\
\hline & Unonopsis lindmanii R.E.Fries & pindaíva-preta & $\mathrm{J}, \mathrm{I}, \mathrm{M}$ & 3523 \\
\hline Apocynaceae & Tabernaemontana catharinensis A. DC. & leiteiro & J, I, M & 3973 \\
\hline Boraginaceae & Cordia sellowiana Cham. & louro & $\mathrm{J}, \mathrm{M}$ & 3979 \\
\hline Burseraceae & Protium heptaphyllum (Aubl.) March. & almécega & J & 3975 \\
\hline Caesalpiniaceae & Peltophorum dubium (Spreng.) Taub. & canafístula & $\mathrm{J}, \mathrm{I}, \mathrm{M}$ & 3974 \\
\hline Cecropiaceae & Cecropia pachystachya Trec. & embaúba & $\mathrm{J}, \mathrm{I}, \mathrm{M}$ & 3569 \\
\hline Clusiaceae & Garcinia brasiliensis Mart. & guanandi & $\mathrm{J}, \mathrm{M}$ & 3982 \\
\hline \multirow[t]{2}{*}{ Elaeocarpaceae } & Sloanea garckeana K. Schum. & carrapateiro & M & 3997 \\
\hline & S. guianensis Vell. & pateiro & $\mathrm{J}, \mathrm{I}, \mathrm{M}$ & 3977 \\
\hline \multirow[t]{2}{*}{ Euphorbiaceae } & Alchornea glandulosa Poeppig & boleiro & $\mathrm{J}$ & 3972 \\
\hline & Croton urucurana Baill. & sangra-d'água & $\mathrm{I}, \mathrm{M}$ & 4004 \\
\hline Fabaceae & Pterocarpus rohrii Vahl. & & J & 3526 \\
\hline \multirow[t]{2}{*}{ Flacourtiaceae } & Casearia gossypiosperma Briquet & espeteiro & $\mathrm{J}, \mathrm{M}$ & 3522 \\
\hline & Xilosma venosum N.E. Brown & espinheiro & I & 3530 \\
\hline Hippocrateaceae & Salacia grandiflora (Mant.) G. Don & rufăo & J, I & 3980 \\
\hline \multirow[t]{5}{*}{ Lauraceae } & Nectandra cuspidata Nees & canela & M & 3971 \\
\hline & N. cissiflora Nees & canelão & $\mathrm{J}, \mathrm{M}$ & 3540 \\
\hline & N. falciflolia (Nees) Castiglioni ex Mez & canelinha & $\mathrm{I}, \mathrm{M}$ & 3571 \\
\hline & N. mollis (HBK) Nees & canela & M & 3539 \\
\hline & Ocotea diospyrifolia (Meissn.) Mez & canela & $\mathrm{J}, \mathrm{I}, \mathrm{M}$ & 3970 \\
\hline \multirow[t]{2}{*}{ Meliaceae } & Guarea guidonia (L.) Sleumer & ataúba & $\mathrm{J}, \mathrm{M}$ & 3994 \\
\hline & Trichilia pallida $\mathrm{Sw}$. & catiguá & $\mathrm{J}, \mathrm{M}$ & 3981 \\
\hline \multirow[t]{4}{*}{ Mimosaceae } & Albizia hassleri (Chodat) Burr. & farinha-seca & $\mathrm{J}, \mathrm{I}, \mathrm{M}$ & 3557 \\
\hline & Inga uruguensis Hook. \& Arn. & ingá & $\mathrm{J}, \mathrm{I}, \mathrm{M}$ & 3968 \\
\hline & 1. laurina (Sw.) Willd. & ingá-branco & $\mathrm{J}, \mathrm{I}$ & 3983 \\
\hline & Zygia cauliflora (Willd.) Killip. & amarelinho & $\mathrm{J}, \mathrm{I}, \mathrm{M}$ & 3574 \\
\hline \multirow[t]{2}{*}{ Moraceae } & Ficus obtusiuscula (Niq.) Miq. & figueira & M & 3575 \\
\hline & Maclura tinctoria (L.) Engl. & taúva & 1 & 3550 \\
\hline \multirow[t]{5}{*}{ Myrtaceae } & Eugenia egensis DC. & & J & 3542 \\
\hline & E. florida DC. & jamelão-do-campo & $\mathrm{J}, \mathrm{I}, \mathrm{M}$ & 4000 \\
\hline & Hexachlamys itatiaiae Mattos & & 1 & 3534 \\
\hline & Psidium guayava $\mathrm{L}$. & goiabeira & $\mathrm{J}, \mathrm{I}$ & 3969 \\
\hline & P. cf. guineense $\mathrm{Sw}$. & goiabinha & $\mathrm{J}$ & 3543 \\
\hline Poaceae & Chusquea sellowii Rupr. & bambu & J, I & 3700 \\
\hline Piperaceae & Piper tuberculatum Jacq. & jaborandi & $\mathrm{J}, \mathrm{M}$ & 4001 \\
\hline \multirow[t]{2}{*}{ Polygonaceae } & Ruprechtia laxiflora Meissn. & marmeleiro & $\mathrm{J}$ & 3984 \\
\hline & Triplaris americana $\mathrm{L}$ & formigueiro & $\mathrm{J}, \mathrm{I}, \mathrm{M}$ & 3985 \\
\hline Phytolacaceae & Galesia integrifolia (Spreng.) Harms. & pau-d'álho & $\mathrm{J}, \mathrm{M}$ & 3986 \\
\hline Rhamnaceae & Colubrina retusa (Pittier) Cowan & & $\mathrm{I}, \mathrm{M}$ & 3996 \\
\hline \multirow[t]{4}{*}{ Rubiaceae } & Coussarea platyphylla. Müll. Arg. & jasmim-da-mata & $\mathrm{J}, \mathrm{M}$ & 3988 \\
\hline & Machaonia brasiliensis (Hoff. \& Humb.) & & & \\
\hline & Cham. et Schl. & & I & 3549 \\
\hline & Psychotria carthagenensis Jacq. & café-de-bugre & M & 4002 \\
\hline Rutaceae & Citrus aurantium $\mathrm{L}$. & laranja-do-mato & $\mathrm{J}$ & 3527 \\
\hline \multirow[t]{2}{*}{ Sapindaceae } & Allophyllus edulis (St. Hil.) Radlk. & vacum & $\mathrm{J}, \mathrm{I}, \mathrm{M}$ & 3989 \\
\hline & Sapindus saponaria $\mathrm{L}$. & sabonete-de-soldado & $\mathrm{J}, \mathrm{I}, \mathrm{M}$ & 3990 \\
\hline
\end{tabular}


Tabela 2. (continuação)

\begin{tabular}{|c|c|c|c|c|}
\hline Famílias & Nome científico & Nome vulgar & Local & HUM \\
\hline \multirow[t]{2}{*}{ Sapotaceae } & Pouteria glomerata (Miq.) Radlk. & maçã-de-pacu & J, I & 3987 \\
\hline & P. torta (Mart.) Radlk. & grão-de-onça & $\mathrm{J}, \mathrm{M}$ & 3995 \\
\hline Sterculiaceae & Guazuma ulmifolia Lam. & mutambo & J, I, M & 3976 \\
\hline \multirow[t]{2}{*}{ Ulmaceae } & Celtis iguanaea (Jaef.) Sargent. & sarã & $\mathrm{M}$ & 3582 \\
\hline & Trema micrantha (L.) Blum & candiúva & $\mathbf{J}$ & 4003 \\
\hline Verbenaceae & Vitex montevidensis Cham. & tarumã & $\mathrm{J}, \mathrm{I}$ & 3992 \\
\hline Indeterminada 1 & & & M & 3524 \\
\hline Indeterminada 2 & & & $\mathrm{M}$ & 3538 \\
\hline
\end{tabular}

elevados para essa região. Souza et al. (1997) assinalam entre $13 \%$ e $54 \%$, numa comparação entre seis levantamentos de ambas as margens e ilhas do rio Paraná. Comparando-se o presente estudo com aqueles avaliados por Souza et al. (1997) e por Campos (1997), obtém-se valores entre $24 \%$ e $61 \%$. Similaridade mais elevada seria esperada para este estudo, tendo-se em vista a homogeneidade topográfica e pedológica observadas, além da proximidade entre os remanescentes.

O Índice de Diversidade de Shannon (H') de 3,20 para a área total constitui o maior valor já assinalado em levantamentos das florestas ripárias do rio Paraná e de seus tributários, nessa região (Souza et al. 1997). Comparando-se com outras regiões, constitui-se, entretanto, num valor dentro da média para florestas ripárias (Bertoni 1984; Rodrigues 1991; Soares-Silva et al. 1992; Kawakita 1994; Felfili 1994; Oliveira et al. 1994; Salis 1994; Carvalho et al. 1995; Chagas e Silva et al. 1995; Nakajima et al. 1996). Analisando-se separadamente os três remanescentes do presente estudo, obtém-se valores entre 2,52 e 2,94 (Tab. 4), sendo estes relativamente baixos para florestas ripárias e dentro da média para essa região do rio Paraná (Souza et al. 1997).

Índices de diversidade baixos para matas ciliares são esperados quando essas são comparadas às matas de planalto e atlântica (Leitão-Filho 1982), sendo que os mais elevados são encontrados em áreas de topografia acidentada e com flutuação não padronizada do lençol freático (Oliveira et al. 1994), o que não é o caso deste estudo. Burkart (1957) e Neiff (1990) assinalam valores baixos para áreas ripárias da planície de inundação do rio Paraná em território argentino e consideram que esses não indicam, necessariamente, que o sistema esteja descompensado, em tensão ou que se encontra em fase imatura de uma série ecológica. Os baixos valores encontrados no presente trabalho podem representar uma resposta ao sistema de cheia e vazante dessa planície, que condiciona a ocorrência de número menor de espécies adaptadas a esse sistema hidrológico.

A análise comparativa dos parâmetros fitossociológicos (Tab. 3) mostra que, dentre as espécies de maior valor de importância (VI), somente Cecropia pachystachya apresentou elevado valor para todos os casos, ocupando a primeira posição nos remanescentes intermediário e montante e a terceira no jusante. Inga uruguensis, embora com menor destaque, encontra-se também entre as mais importantes nos três remanescentes, possuindo, entretanto, melhor colocação no intermediário. Essas duas espécies heliófitas, consideradas generalistas, estão presentes em todos os levantamentos fitossociológicos comparados por Souza et al. (1997), para essa região de estudo apresentando, ainda, ampla distribuição em matas ciliares do Estado de São Paulo (Barbosa 1993).

Unonopsis lindmanii, espécie ombrófila que ultrapassa o VI de Cecropia pachystachya no remanescente a jusante, encontra-se bem representada também a montante, enquanto que no 


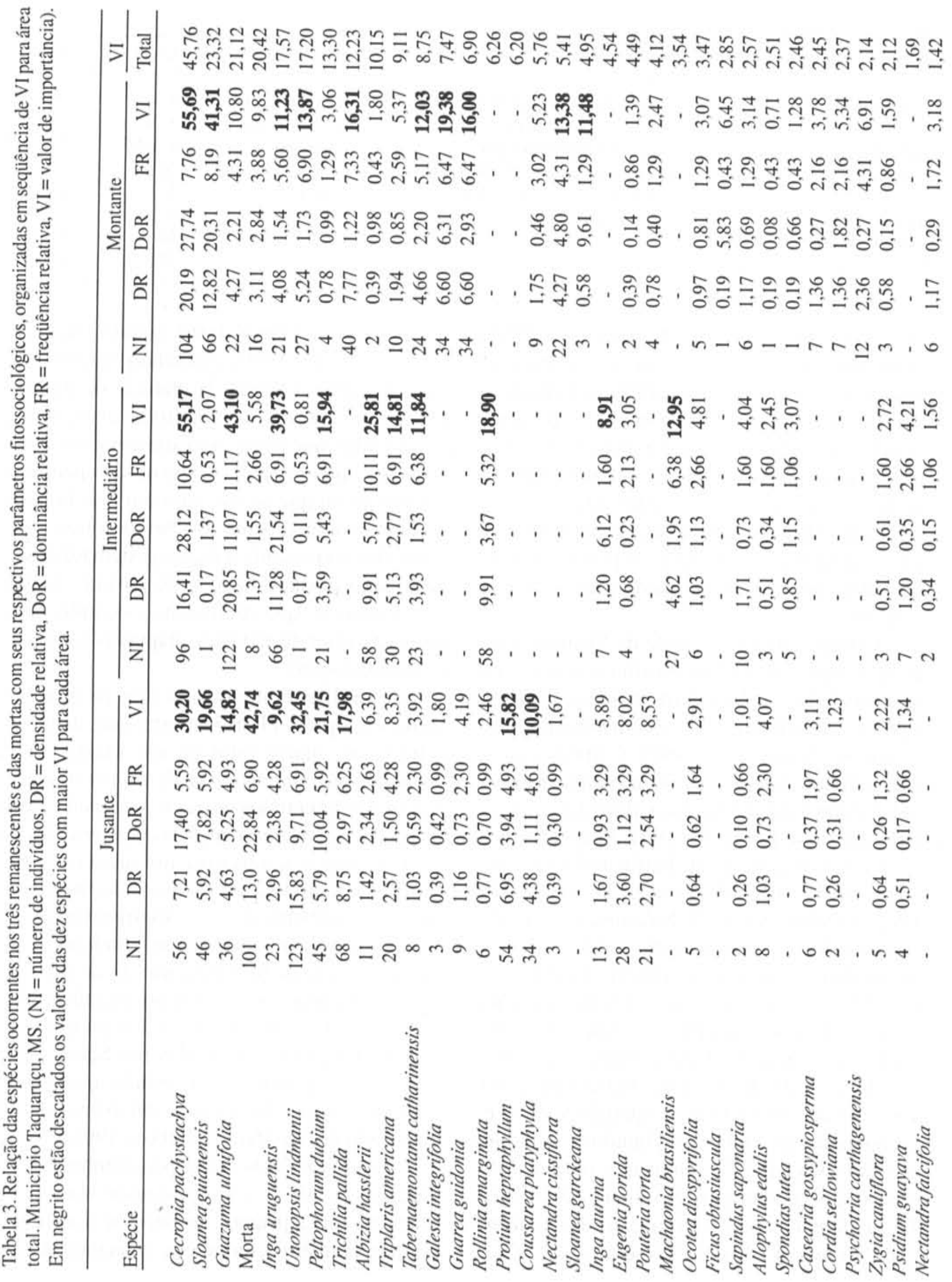




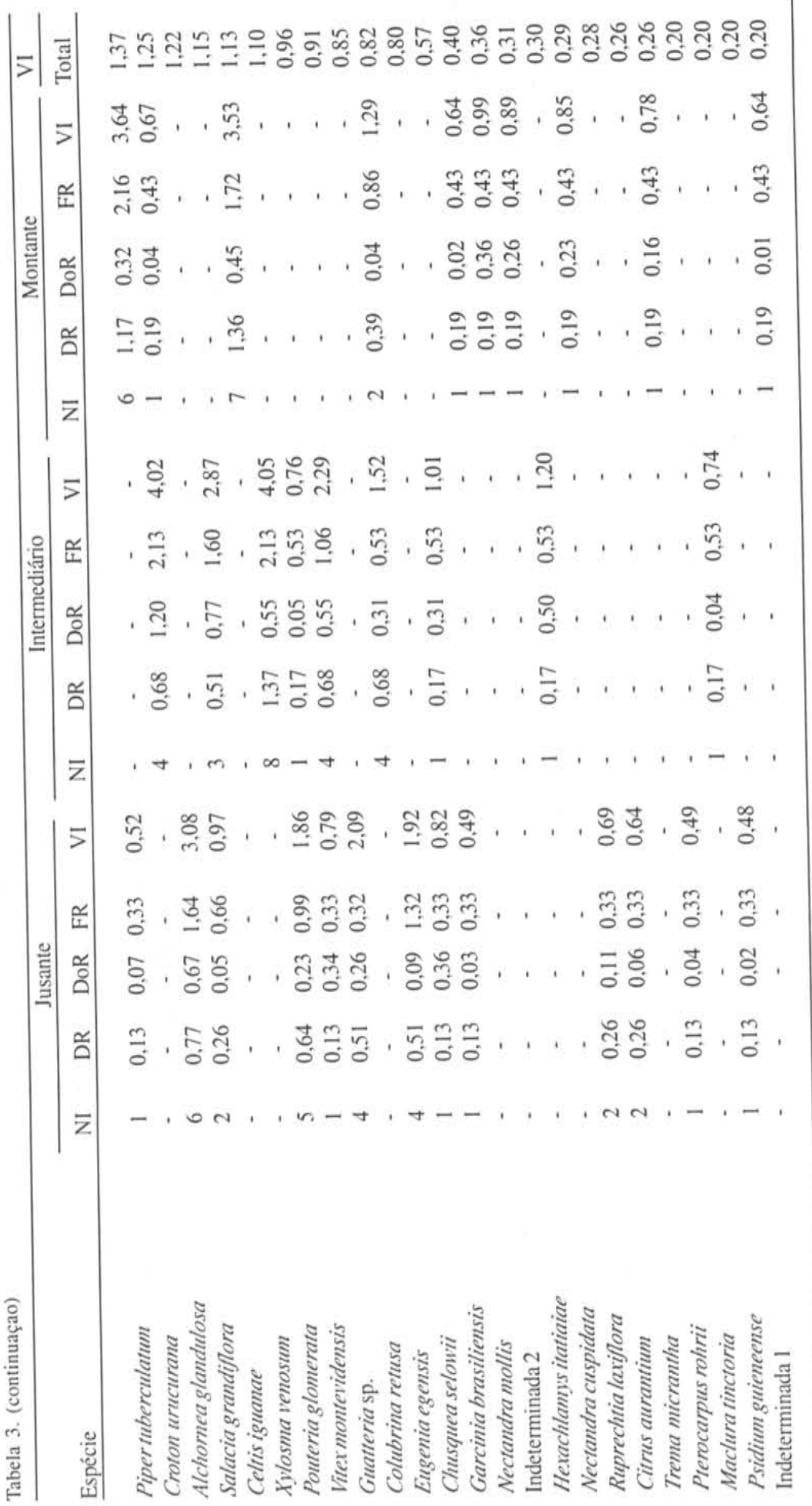


intermediário ocupa a $28^{\text {a }}$ colocação. Sloanea guianensis, Guarea guidonia e Trichilia pallida, consideradas como típicas de estádios avançados de sucessão (Budowski 1965; Smith \& Smith 1970; Kageyama 1992; Mantovani 1993), encontram-se, também, bem representadas apenas nos remanescentes montante e jusante.

Os indivíduos mortos possuem diferentes VI entre os três remanescentes, sendo de 42,74 para jusante, 9,83 para montante e 5,58 para o intermediário. Elevados índices, como o encontrado no remanescente a jusante, são relacionados por Assis (1991) e Salis (1990) com períodos de inundação acima da média e com ações antrópicas.

As diferenças florísticas e fitossociológicas, entre esses remanescentes são reforçadas quando se analisam os diversos parâmetros estruturais (Tab. 4), tais como número de indivíduos, espécies e famílias, percentagem de plantas mortas, índice de diversidade, densidade total e área basal, podendo-se observar que o remanescente a jusante reúne os maiores valores, com exceção da área basal, sendo, então ultrapassado pelo montante. Esta alteração no padrão de resposta está relacionada à presença, a montante, de espécies que possuem elevado diâmetro, tais como Sloanea guianensis, Sloanea garckeana e Ficus obtusiuscula, enquanto o trecho intermediário, que apresenta o menor valor para esse mesmo parâmetro, possui muitos indivíduos com diâmetro relativamente pequeno, tais como Guazuma ulmifolia, Albizia hassleri, Rollinia emarginata e Machaonia brasiliensis, espécies essas com características de estádios iniciais de sucessão.

Para o remanescente intermediário, os resultados acima apresentados e somados à menor riqueza florística, menor índice de diversidade (H') (Tab.4) e melhor representatividade de espécies de estádios iniciais de sucessão, sugerem perturbações antrópicas mais recentes, em relação aos outros dois.

$\mathrm{O}$ remanescente a jusante, um dos que se encontram em estádio mais avançado de sucessão, de acordo com as considerações acima, apresenta também o maior VI para as árvores mortas, grupo este constituído principalmente por indivíduos da pioneira Cecropia pachystachya (Budowski 1965; Kageyama 1992; Mantovani 1993), que no processo de sucessão estaria perdendo a vitalidade e sendo substituída por espécies de categorias sucessionais mais avançadas, como Unonopsis lindimanii, a segunda colocada em VI.

Tendo-se em vista os resultados obtidos quanto à topografia e ao solo, acredita-se que antropismos pretéritos, freqüentes nessa região, possam ter funcionado como um dos principais fatores da estrutura atual desses três remanescentes.

As diferentes características fitossociológicas encontradas em áreas tão próximas reforça a necessidade da multiplicação deste tipo de investigação para outros remanescentes florestais dessa planície, ampliando-se, assim, o conhecimento dessas formações e servindo como instrumento útil na avaliação e planejamento de ações de recuperação ambiental.

Tabela 4. Dados estruturais e de diversidade de espécies referentes ao levantamento fitossociológico para indivíduos com PAP $\geq 15 \mathrm{~cm}$ dos três remanescentes. Município Taquaruçu, $\mathrm{MS}$. (NI = número de indivíduos; $\mathrm{NE}=$ número de espécies, $\mathrm{H}^{\prime}(\mathrm{esp})=$ índice de diversidade de Shannon para espécies, $\mathrm{NF}=$ número de famílias, $\mathrm{H}^{\prime}(\mathrm{fam})=$ índice de diversidade de Shannon para famílias, $\mathrm{DT}=$ densidade total, $\mathrm{AB}=$ área basal total, $\mathrm{IM}=$ indivíduos mortos).

\begin{tabular}{lrllllllr}
\hline LOCAL & NI & NE & H'(esp) $^{\prime}$ & NF & H'(fam) $^{\prime}$ & DT/ha & AB/ha & IM (\%) \\
\hline Jusante & 777 & 40 & 2,94 & 25 & 2,69 & $2.466,67$ & 32,04 & 14,3 \\
Intermediário & 585 & 29 & 2,52 & 20 & 2,28 & $1.857,14$ & 22,69 & 1,9 \\
Montante & 515 & 37 & 2,88 & 25 & 2,61 & $1.634,92$ & 45,81 & 3,3 \\
Área total & 1877 & 56 & 3,20 & 30 & 2,80 & $1.986,24$ & 33,51 & 7,0 \\
\hline
\end{tabular}




\section{Referências bibliográficas}

Agostinho, A. A. \& Zalewski, M. 1996. A planície alagável do alto rio Paraná: importância e preservação. Editora da Universidade Estadual de Maringá, Maringá.

Assis, M. A. 1991. Fitossociologia de um remanescente florestal do rio Ivinheima (MS). Dissertação de Mestrado. Universidade Estadual de Campinas, Campinas.

Barbosa, L. M. 1993. Vegetação ciliar: conceitos e informações práticas para conhecer e recuperar remanescentes degradados. Caderno de Pesquisa. Série Botânica 1: 3-36.

Bertoni, J. E. A. 1984. Composição florística e estrutura fitossociológica de uma floresta do interior do Estado de São Paulo: Reserva Estadual de Porto Ferreira, SP. Dissertação de Mestrado. Universidade Estadual de Campinas, Campinas.

Budowski, G. 1965. Distribution of tropical Americam forest species in a light of successional processes. Turrialba 15(1): 40-42.

Bukart, A. 1957. La vegetación del delta del Rio Paraná. Darwiniana 11(3): 457-563.

Campos, J. B. \& Souza, M. C. 1997. Vegetação. Pp. 333-344. In A. E. A. de M. Vazzoler, A. A. Agostinho \& N. S. Hahn (Eds.), A planície de inundação do alto rio Paraná: aspectos físicos, biológicos e socioeconômicos. Editora da Universidade Estadual de Maringá. Maringá.

Campos, J. B.; Romagnolo, M. B.; Souza, M. C. 1997. Structure composition and, spacial distribuition, tree species in a remnant of the semideciduous seasonal alluvial forest of the upper Parana river floodplain. Brazilian Archives of Biology and Technology 43(2): no prelo.

Carvalho, D. A.; Oliveira-Filho, A. T.; Vilela, E. A. \& Gavilanes, M. L. 1995. Flora arbustivo-arbórea de uma floresta ripária no alto rio Grande em Bom Sucesso/MG. Acta Botanica Brasilica 9(2): 231-245.

Chagas e Silva, F.; Fonseca, E. P.; Soares-Silva, L. H.; Müller, C. \& Bianchini, E. 1995. Composição florística e fitossociologia do componente arbóreo das florestas ciliares da bacia do rio Tibagi-3. Fazenda Bom Sucesso, Município de Sapopema, PR. Acta Botanica Brasilica 9(2): 289-302.

Elmore W. 1992. Riparian responses to grazing practices. Pp. 442-457. In: R. J. Naiman (Ed.), Watershed management: balancing sustainability and enviromental change. SpringerVerlag, New York.
EMBRAPA (Empresa Brasileira de Pesquisa Agropecuária). 1984. Levantamento de reconhecimento dos solos do Estado do Paraná. t.2. (Boletim técnico), Curitiba.

Felfili, J. M. 1994. Floristic composition and phytosociology of the gallery forest alongside the Gama stream in Brasília, DF, Brazil. Revista Brasileira de Botânica 17(2): 1-11.

Fidalgo, O. \& Bononi, V. L. R. 1989. Técnicas de coleta, preservação e herborização de material botânico. Instituto de Botânica/Secretária do Meio Ambiente, São Paulo.

Godoy, R. 1988. Topografia básica. Fundação de Estudos Agrários Luiz de Queiroz ESALQ/FEALQ, Piracicaba.

Holland, M. M. \& Risser, P. G. 1988. Introduction: the role of landscape boundaries in the management and restoration of changing environments. Pp. 1-8. In M. M. Holland, P. G. \& R. J. Naiama (Eds.), Ecotone. Chapman and Hall, London.

IAPAR - Instituto Agronômico do Paraná, Curitiba. 1994. Cartas climáticas do Estado do Paraná. IAPAR, Londrina.

Johnson, R. A. \& Wichern, D. W. 1992. Appliede multivariate statistical analysis. Prentice Hall International Editors, London.

Kageyama, P.Y. 1992. Recomposição da vegetação com espécies arbóreas nativas em reservatórios de usinas hidrelétricas da CESP. Piracicaba. Série Técnica IPEF, 8(25): 1-43.

Kawakita, K. 1995. Florística e estrutura fitossociológica de um trecho da floresta ciliar da margem esquerda do ribeirão dos Apertados, Parque Estadual Mata dos Godoy, Londrina, PR. Monografia de Bacharelado. Universidade Estadual de Londrina, Londrina.

Leitão-Filho, H.F. 1982. Apectos taxonômicos das florestas do Estado de São Paulo. Anais do Congresso Nacional de Essências Nativas, Campos do Jordão. Silvicultura em São Paulo 16A: 197-206.

Mantovani, W. 1989. Conceituação e fatores condicionantes. Pp.11-19. In: L. M. Barbosa (Coord.), Anais do Simpósio sobre Mata Ciliar. Fundação Cargil, Campinas.

Mantovani, W. 1993. Estrutura e dinâmica da Floresta Atlântica na Juréia, Iguapé-SP. Tese de Doutorado. Universidade de São Paulo, São Paulo.

Martins, F. R. 1979. O método de quadrantes e a fitossociologia de uma floresta residual do interior do Estado de São Paulo. Tese de Doutorado. Universidade de São Paulo, São Paulo. 
Matteucci, S. D. \& Colma, A. 1982. Metodologia para el estudio de la vegetacion. Secretaria General de la Organizacion de los Estados Americanos. Programa Regional de Desarrollo Cientificio y Tecnológico, Washington.

Müller-Dombois, D. \& Ellemberg, H. 1974. Aims and methods of vegetation ecology. John Wiley \& Sons, New York.

Nakajima, J. N.; Soares-Silva, L. H.; Medri, M. E.; Goldemberg, R. \& Correa, G.T. 1996. Composição florística e fitossociológica do componente arbóreo das florestas ripárias da Bacia do rio Tibagi: 5. Fazenda Monte Alegre, Município de Telêmaco Borba, Paraná. Arquivo de Biologia e Tecnologia 39(4): 933-948.

Neiff, J. J. 1990. Ideas para la interpretacion ecologica del Paraná. Interciencia 15(6): 424-441.

Oliveira-Filho, A. T.; Almeida, R. J.; Mello, J. M. \& Gavilanes, M. L. 1994. Estrutura fitossociológica e variáveis ambientais em um trecho da mata ciliar do córrego dos Vilas-Boas, Reserva Biológica do Poço Bonito, Lavras (MG). Revista Brasileira de Botânica 17(1): 67-85.

Rodrigues, R. R. 1989. Análise estrutural das formações florestais ripárias. Pp.99-119. In: L. M. Barbosa (Coord.), Anais do Simpósio sobre Mata Ciliar. Fundação Cargil, Campinas.

Rodrigues, R. R. 1991. Análise de um remanescente de vegetação natural às margens do rio Passa Cinco, Ipeúna, SP. Tese de Doutorado. Universidade Estadual de Campinas, Campinas.

Romagnolo, M. B.; Souza-Stevaux, M. C. \& Ferrucci, M. S. 1994. Sapindaceae da planície de inundação do trecho superior do rio Paraná. Revista Unimar 16(3): 61-81.

Salis, S. M. 1990. Composição florística e estrutura de um remanescente de mata ciliar do rio Jacaré-Pepira, Brotas, SP. Dissertação de Mestrado. Universidade Estadual de Campinas, Campinas.

Silva, P. M. 1995. Polygonaceae da planície de inundação do trecho superior do rio Paraná. Monografia de Bacharelado. Universidade Estadual de Maringá, Maringá.
Smith, C. E. \& Smith, L. B. 1970. Eleocarpáceas. In: P. R. Reitz (Ed.), Flora Ilustrada Catarinense. Itajaí: Herbário Barbosa Rodrigues.

Soares e Silva, L. H.; Bianchini, E.; Fonseca, E. P.; Dias, M. C.; Medri, M. E. \& Zangaro-Filho, W. 1992. Composição florística e fitossociologia do componente arbóreo das florestas ciliares da bacia do rio Tibagi. 1. Fazenda Doralice - Ibiporã, PR. Pp.199-206. In Anais Congresso Nacional sobre Essências Nativas, 2., São Paulo 1992. Instituto Florestal, São Paulo.

Souza, D. C. \& Souza, M. C. 1998. Levantamento da florístico das tribos Psychotrieae, Coussareeae e Morindeae (Rubiaceae) na região de Porto Rico, alto rio Paraná. Acta Scientiarum 20(2): 207-212.

Souza, M. C. 1998. Estrutura e composição florística da vegetação de um remanescente florestal da margem esquerda do rio Paraná (Mata do Araldo, Município de Porto Rico, PR). Tese de Doutorado. Universidade Estadual Paulistra, Rio Claro.

Souza, M. C.; Cislinski, J. \& Romagnolo, M. B. 1997. Levantamento florístico. Pp.345-370. In A. E. A. de M. Vazzoler, A. A. Agostinho \& N. S. Hahn (Eds.), A planície de inundação do alto rio Paraná: aspectos físicos, biológicos e socioeconômicos. Editora da Universidade Estadual de Maringá, Maringá.

Souza-Stevaux, M. C.; Negrelle, R. R. B. \& CittadiniZaneti, V. 1994. Seed dispersal by the fish Pterodoras granulosus in the Paraná River Basin, Brazil. Journal of Tropical Ecology 10(4): 621-626.

Stevaux, J. C. 1994. Geomorfologia, sedimentologia e paleoclimatologia do Alto Curso do rio Paraná (Porto Rico, PR). Boletim Paranaense de Geociências 42: $97-112$.

Veloso, H. P. \& Góes-Filho, L. 1982. Fitogeografía Brasileira - Classificação fisionômico-ecológica da vegetação neotropical. IBGE, Salvador.

Zalewski, M.; Thorpe, J. B. \& Gaudin, P. 1990. Review of the session: rivers. Pp.17-20. In M. Zalewski; J. B. Thorpe; P. Gaudin (Eds.), UNESCO MAB. Fishes and Land/Inland water ecotones. University of Lódz.; University of Stirling; Universite Claude Bernard Lyon I. Lódz, Poland. 